\title{
Protée
}

\section{D’événements en avènements}

\section{La mémoire visionnaire dans Terra Nostra}

\section{Julie Hyland}

Volume 32, numéro 1, printemps 2004

Mémoire et médiations

URI : https://id.erudit.org/iderudit/011025ar

DOI : https://doi.org/10.7202/011025ar

Aller au sommaire du numéro

\section{Éditeur(s)}

Département des arts et lettres - Université du Québec à Chicoutimi

ISSN

0300-3523 (imprimé)

1708-2307 (numérique)

Découvrir la revue

Citer cet article

Hyland, J. (2004). D’événements en avènements : la mémoire visionnaire dans Terra Nostra. Protée, 32(1), 49-58. https://doi.org/10.7202/011025ar
Résumé de l'article

Le roman Terra Nostra de Carlos Fuentes constitue une tentative singulière d'écriture et de représentation des événements du passé de l'Amérique colonisée dans le contexte d'une relation à l'Espagne conquérante. Il se risque à une interprétation moins dépendante des faits que de l'imaginaire. En faisant du rêve son suppôt d'interprétation et de remémoration du passé, Terra Nostra fait aussi du temps une peau de taureau tendue entre les deux aspirations indissociables de la réminiscence et de l'advenir. L'intégration du rêve permet de concevoir une mémoire pluridirectionnelle qui cherche autant le souffle d'un avenir dans le passé que les fruits de germes lointains dans le déploiement de l'actualité. Bien plus qu'une suture subjective qui mêle et adapte l'histoire à la mémoire, le rêve est aussi le moyen par lequel se médiatise la mémoire dans Terra Nostra.
Ce document est protégé par la loi sur le droit d'auteur. L'utilisation des services d'Érudit (y compris la reproduction) est assujettie à sa politique d'utilisation que vous pouvez consulter en ligne.

https://apropos.erudit.org/fr/usagers/politique-dutilisation/ 


\title{
D'ÉVÉNEMENTS EN AVÈNEMENTS: LA MÉMOIRE VISIONNAIRE DANS TERRA NOSTRA
}

JULIE HYLAND

\author{
Peuples! écoutez le poète! \\ Écoutez le rêveur sacré! \\ Dans votre nuit, sans lui complète, \\ Lui seul a le front éclairé. \\ Des temps futurs perçant les ombres, \\ Lui seul distingue en leurs flancs sombres \\ Le germe qui n'est pas éclos. [...] \\ Toute idée, humaine ou divine, \\ Qui prend le passé pour racine, \\ A pour feuillage l'avenir. \\ V. Hugo, Les Rayons et les Ombres, p. 6.
}

Au cœur de Terra Nostra, de l'écrivain mexicain Carlos Fuentes, siège le souvenir confus, mais tenace, d'une anté-Amérique. Toujours niché en amont d'oublis, ce souvenir s'introduit dans les chambres ombreuses du rêve de manière à y survivre. Ayant fait du rêve son support d'interprétation et de remémoration du passé espagnol, Fuentes en est venu à constituer une tentative singulière de représentation des événements de la Conquista. L'intégration du rêve dans la tessiture du texte a permis la conception d'une mémoire multidirectionnelle qui cherche le souffle d'un avenir dans le passé, de même que les fruits de germes lointains dans le déploiement de l'actualité. Terra Nostra a fait du temps une peau de taureau tendue entre les deux aspirations indissociables du ressouvenir et de l'advenir.

De fait, ce qu'expérimentent les différents personnages du roman, ce sont les horizons du passé et du futur rendus indifférenciés par une mémoire visionnaire du rêve. La construction temporelle du roman est ainsi fondée sur des moments d'hypothèses et de désirs, selon lesquels passé et présent se déclinent constamment en un futur, en une recherche d'advenir. De ce point de vue, Terra Nostra accomplit une compréhension imaginaire et affective de l'événement; filé par les survivances d'un présent psychique, son essor herméneutique s'étoile en désirs, souvenirs et imaginations. 


\section{SPECTRES DE TEMPS}

L'univers romanesque, fondé sur une orchestration et une expérimentation du temps fort hybride, permet de réinvestir l'histoire de visions que des sujets ont eues du futur dans le passé: non pas exclusivement de ce qui est advenu, mais aussi de ce qui aurait pu advenir. Le personnage de Domine Valerio, le maître du théâtre de la mémoire, résume l'entreprise du roman par l'idée d'une mémoire simultanée: «regarde, regarde dans les trames combinées de mon théâtre le passage de la plus absolue des mémoires: la mémoire de ce qui aurait pu être et qui ne fut point» (t.II: 121) ${ }^{1}$. Le théâtre de Valerio préserve la tonalité de ce que fut hier. À la différence de l'historien, il tient à concevoir une mémoire qui, au lieu d'être au service de l'événement, aspire à rendre compte de l'avènement. Sa mémoire inventée est de nature «avènementielle», dans la mesure même où son activité de représentation puise de part et d'autre dans l'anté-événementiel et le postévénementiel; aussi bien dire dans le désir. Tout est entrevu «par les trous de l'oubli, par les orifices du temps, dans les obscurs instants de vide durant lesquels le passé tenta d'imaginer le futur» (t.II: 174). Cette mémoire imaginée par le maestro vénitien s'intéresse à la déclivité du fait, c'est-à-dire à l'ubac et à l'adret de la raison pour laquelle, par exemple, Cicéron garda le silence tandis qu'il écoutait les sottises de Catalina (t.II: 121). Valerio observe et analyse les événements du passé sous plusieurs angles, sachant fort bien que toute écriture, décision et situation reposent sur un «interminable choix entre ceci, ceci et cela» (t.II: 121) et que, ce faisant, la détermination historique dépend intimement de ce dont elle se dissocie. Julian, le peintre du roi, affirme également le caractère virtuel de toute réalité. Selon lui, l'œuvre picturale, à l'image du monde, ne se définit pas seulement par sa description objective; elle est aussi composée de tous les possibles qu'elle renferme. Du coup, le rôle des artistes, qu'il s'agisse de celui du peintre, de l'écrivain ou du créateur du théâtre de la mémoire, est entièrement relatif à une capacité imaginative. La réserve d'images dans l'image et de souvenirs dans la mémoire dépend étroitement de l'imagination qui l'insuffle. Ainsi le peintre tient ainsi ces propos au Sire: «Moi je ne vois que ce qui donne vie à l'esprit intangible ainsi qu'à la matière inerte: l'imagination» (t. II: 201). De concours avec l'analyse, l'imagination recrée des liens en tenant compte de ce qui aurait pu être:

Moi Julian, moine et peintre, je te dis que de même que les paroles contradictoires du Seigneur et de Ludovico se confondent pour faire naître un nouveau discours né de la rencontre de deux contraires, de même se combinent ombre et lumière, forme et volume, couleur plane et profondeur de la perspective sur une toile, ainsi devraient se combiner dans ton livre le réel et le virtuel, ce qui fut et ce qui aurait pu être, ce qui est avec ce qui pourrait être. Pourquoi nous conterais-tu seulement ce que nous savons déjà au lieu de nous dévoiler ce que nous ignorons? (t. II: 269)

L'inventeur rappelle à Ludovico l'incidence que peut avoir un infime détail sur un événement qui est toujours passible de prendre une allure tout à fait différente. L'histoire est contingente et elle se répète, nous apprend-il encore, «seulement parce que nous ignorons l'autre réalité possible de chaque fait historique" (t. II : 123). Il existerait ainsi, parallèlement à des séries réelles de faits, des séries idéales permettant au passé d'être ce qu'il est par distinction de ce qu'il aurait pu être. En fait, et c'est sur ce point que nous pouvons faire le lien avec la question du temps dans le roman, l'invention de Domine Valerio est formellement visionnaire, car elle propose ce qui aurait pu être et qui n'a pas eu lieu de manière effective, comme un modèle de ce qui est susceptible d'advenir. Comprendre et mettre en lumière les rejets de l'histoire, c'est conséquemment aller dans le sens de son avènement. C'est pourquoi, dans l'œuvre de Fuentes, l'avenir se déduit du passé et que, d'une autre manière, le rêve induit la mémoire:

Les images de mon théâtre intègrent toutes les possibilités $d u$ futur, car en sachant ce qui ne fut pas, nous saurons ce qui brûle d'être: ce qui a été - tu l'as vu - est un fait latent qui attend le moment d'être, sa seconde chance. (Ibid.)

Or, à la considérer sous l'angle d'une latence, l'histoire fuentésienne, pour reprendre les mots du grand Monarque, trace tout « un futur de 
résurrections» (t.II: 174). Car c'est ce qui a été caché, rejeté ou encore tué qui refait surface dans le roman: «les gestes non accomplis, les paroles imprononcées, les choix sacrifiés, les décisions toujours reportées» (t. II: 121). Passionnée d'anecdotes sises hors de l'histoire (d'absences), la mémoire fuentésienne mène à deux constats. Le premier est que ne cesse jamais directement d'exister ce qui a été oublié et, l'autre, qu'il y a de l'inconscient dans l'histoire, c'est-à-dire que la connaissance du passé, toujours incomplète dans ses sources, objets et compréhension, est toujours insuffisante et oublieuse à l'égard de ce qu'elle représente. Le discours historique, ou de tout autre nature qu'il soit, est toujours au bord d'autre chose: il se ferme et se coupe d'éléments de manière à ce que sa situation puisse se laisser représenter comme l'envers et l'endroit d'une bande de Möbius. Pour chaque écho, le roman dévoile ainsi une mémoire écrite et instituée à l'envers de laquelle s'en trouve une seconde de nature hiéroglyphique, c'est-à-dire encore indéchiffrable.

\section{OCCUPATION TEMPORELLE: L'INDÉCHIFFRABLE}

Le présent de la narration - qui se situe vraisemblablement dans l'épilogue vibrant du vingtième siècle parisien - présente deux protagonistes, Célestine et Pollo, dont chacun des propos est marqué par le stigmate d'une violence passée. La situation initiale du livre, "Chair, sphères, regard gris au bord de la Seine», est dévastée par un mal dont la cause est indiscernable. Les femmes accouchent par milliers sur les rives du fleuve, les gens s'entretuent inopinément sur les grandes avenues, et la ville lumière est intoxiquée par un miasme intolérable, «comme si l'enfer avait déchargé toute la congestion de ses poumons» (t. I: 19); mais personne au sein de cette tragédie ne semble comprendre la raison du bouleversement. Est-ce, comme s'interroge Pollo, «cause, effet, problème ou solution...» (t. I : 29)? Bien que d'innombrables groupes avancent dans les rues encombrées de Paris en scandant cette ritournelle obsédante: «Le lieu c'est ici/Le temps maintenant/ Maintenant et ici/ Ici et maintenant» (ibid.), tout porte néanmoins à croire que le témoignage d'instants antérieurs persévère et subsiste en ce moment de catastrophe; s'y jouent là les effets d'une durée exprimée par le détour de survivances et de survenances. À la première ritournelle, qui valorise seulement le lieu et le temps de l'énonciation, conviendrait davantage une autre rengaine. Cette dernière accorderait un lieu qui, même ici, n'en serait pas moins habité par un temps passé:

Les envahisseurs ne sont pas venus d'un autre lieu mais d'un autre temps, se dit finalement Pollo. L'antimatière qui a rempli les vides de ton présent a été engendrée et stockée dans le passé.

Le roman apparaît ainsi comme une survivance du passé hispanique. Il est à la fois processus et effets: manifestation de traces et d'un travail du temps.

L'importance accordée au futur dans le roman est proportionnelle à l'insignifiance attribuée à divers pans du passé. L'introduction dramatique et pléthorique insiste, non seulement sur ce qui a été perdu, mais sur le fait que plusieurs éléments et personnes ont disparu dans les déserts sans écritures de l'oubli. Ainsi, le premier chapitre revient comme un mal négligé sur certains événements du passé. Le fil semble rompu par la violence d'un aboutissement, car il y a une difficulté à savoir à quelle fin exactement répond cet événement. À l'instar des personnages qui accomplissent cet épisode, le lecteur peut se demander: "mais que s'est-il passé pour en arriver à ça?». La vision angoissée du futur qui préfigure une apocalypse construit une véritable intrigue, car elle demande pourquoi à cette représentation traumatisée qui poursuit une limite. Elle pose, en effet, toute la question du passé.

Le rapport du futur au passé, dans le monde romanesque de Fuentes, est médiatisé et structuré par une mémoire de la Conquista qui agit comme creux et crête. Le blocage des forces du passé, leur immaturation est, en ce sens, responsable «de la répétition anachronique et verbeuse du passé " ${ }^{2}$ qui sévit dans la ville parisienne. Le délai, voire l'obstacle rencontré dans l'accomplissement des désirs, 
occasionne un récit anachronique, sorte de spectre qui réapparaît toujours sous la forme d'une lacune et d'une dette appelées à être exaucées et acquittées dans un futur qui s'offre comme un horizon de potentialités latentes: "Ce transfert de passé [est] celui de la moins réalisée, la plus avortée, la plus latente et désirante de toutes les histoires: celle de l'Espagne et de l'Amérique espagnole» (t. II: 453). Cette disposition anachronique implique de reconnaitre, dans la dynamique temporelle du roman, un principe d'animation pensable et traduisible en termes psychiques, en termes de refoulement et d'inconscient. Car en raison d'offenses passées, il y a quelque chose qui ne peut être tu, et qui de ce fait signe un parcours récursif et tenace. Sans convocation ni raison, le monde ruiné par la colonisation espagnole revient sous la forme répétitive d'un une fois encore dans le Paris du XXe siècle. Le roman et tous les personnages qu'il implique vivent alors au gré de battements rythmiques qui font sans cesse appel à la mémoire d'une autre époque. De ce point de vue, à partir du deuxième chapitre, l'Espagne du siècle d'or s'offre comme une explication et une conséquence du désastre contemporain de la narration qui renvoie à l'image, au rappel d'un premier désastre génocidaire: à l'espace lointain et oublié de l'Amérique. La survivance ${ }^{3}$, en tant que rappel du passé, détermine la nature étrange des liens qui se nouent entre les époques et les espaces. Aussi le roman présente-t-il la ville de Paris comme homologue de l'Espagne de Philippe II, ou du moins comme sa translation: s'y réverbèrent effectivement de nombreux événements du siècle d'or espagnol. Les Parisiens subissent de façon effrayée et stupéfaite le retour d'un passé étranger à leur histoire. Cette situation suggère une sorte de mise à l'épreuve des Parisiens qui se trouvent contrariés dans leur intimité par un mal qui ne les concerne pas, qui leur vient du dehors. Ils sont victimes d'un décalage et se trouvent manipulés par un temps en rupture évidente avec le leur. Cette interversion du temps dans l'espace parisien se lit comme une forme de terrorisme temporel, où le passé espagnol vient précisément occuper, accaparer et terrifier le présent parisien:
[...] nous n'avons pas été envahis par les Martiens ou les Vénusiens, mais par les momies et les hérétiques du XVe siècle, les conquérants et les peintres $d u$ XVI siècle, [...]: nous avons été occupés par le passé. (t. II: 453)

\section{PASSÉ PAR LE RÊVE}

Les transferts spatio-temporels s'effectuent par le biais du rêve. En effet, Terra Nostra assigne au rêveur une fonction de passeur: il est un transmetteur d'images et de mémoires. Il est l'être du passage entre divers lieux, temps et états. Il sert résolument de lien. Le rêveur fuentésien fonctionne à rebrousse-poil, par micro-lectures; tel est le sens de son activité, de sa figuration: partir d'un élément contemporain pour l'approfondir et l'investir dans le dessein de rejoindre un originaire. Ainsi, même si l'action de Terra Nostra débute dans un Paris du XXe siècle rempli de divers symptômes et trous de mémoire, elle tend néanmoins à rejoindre progressivement la cause de tant de réactions; elle renvoie à l'Espagne de la conquête, à ce vers quoi elle fait signe. La mémoire, qui structure et réalise l'ensemble des rapports narratifs dans le roman, est inspirée généralement par une démarche analytique constituée de liens à valeur rétrospective. C'est pourquoi, à la suite de l'introduction qui se déroule au XXe siècle à Paris, le roman poursuit son action en faisant refouler un personnage sur les terres espagnoles, au XVI ${ }^{\mathrm{e}}$ siècle. On pourrait dire, dans une certaine mesure, que le récit résulte d'une interprétation des différentes empreintes laissées dans un Paris assailli par une énigme qui le laisse à l'agonie. Comme si l'insoutenable même de la situation parisienne inspirait ou marquait à ce point les personnages de Pollo et de Célestine que ceux-ci devaient se prêter à un processus "profond" d'interrogation et d'analyse. Conséquemment, la suite du premier chapitre se pose comme une plongée dans des eaux qui, loin de faire apparaître une issue, creusent des entrées dans des antériorités, dans des histoires passées. En ce sens, la mémoire fuentésienne apparaît comme une opération sémantique; répondant aux conditions de la régression et du doute, elle disperse et fait voler en éclats des blocs 
d'histoire afin d'en produire d'autres. Elle reste toujours, en définitive, une mise en relation avec le passé qui fait refluer non seulement des faits, mais surtout des affects, et c'est pourquoi elle se laisse davantage penser comme le siège d'investissements émotifs qui assure la pérennité des traces mnésiques.

\section{LA CRYPTE DU RÊVE}

Je me souvins alors de mon rêve - celui où je creusais un puits pareil à celui-ci et tombais dedans...

Carlos Fuentes, Terra Nostra, t.I: 640.

Dans Terra Nostra, le rêveur médiatise l'accès à une mémoire lointaine et dissimulée, il en règle l'évocation de façon évidente et particulière. Le narrateur dit ainsi «que le Seigneur retrouve dans son sommeil le souvenir" (t.I: 76) et Célestine poursuit un peu plus loin dans le roman en tenant ces propos révélateurs: «En te rêvant je te faisais surgir de ma mémoire» (t. I: 261). Pour Célestine, il n'y a pas de frontière entre rêve et mémoire: «Mère du fils du charpentier, je me souviens de tout comme d'un rêve" (t.I: 311). Le rêve, en effet, l'oriente vers la mémoire, l'amène à se souvenir d'images et d'impressions muettes dans la vie vigile. Sa mémoire en dépend, car le fait de rêver lui esquisse une expérience de la réminiscence du monde fort distante des commémorations mises en place par une intellection du temps.

La situation singulière du roman, par laquelle le rêve restitue plusieurs événements du passé à la surface du texte, présente l'expérience onirique comme l'un des derniers supports mnésiques permettant de fouiller les dépôts et les alluvions laissés par l'histoire. Chaque fait est ainsi rappelé à l'ordre du discours dans un désordre activé par la dynamique onirique. Tout revient, et même plus encore que ce qui pouvait se laisser entendre, mais tamisé par le point de vue récréatif du rêve. Les sédiments mnésiques, rejetés des volcans de l'oubli et d'une forme de nomination, refluent alors dans une disposition surprenante. Le rêveur fuentésien interroge et dénoue les liens entre les événements et, ce faisant, il peut apparaître délirant, justement parce qu'au lieu de présenter une organisation logique, c'est-à-dire normalisée, il s'attache à créer de nouvelles liaisons. Il présente le flux de ses expériences tel qu'il le ressent, c'est-à-dire d'une façon beaucoup plus pulsionnelle et imaginative que rationnelle. Il ne s'attarde pas à une éthique qui chercherait l'ordre et la vérité de l'énoncé. Son expérience n'est ainsi ni restrictive ni réflexive, mais plutôt intensive, spontanée et spéculative. De fait, l'ensemble des signes arrangés en légende par le discours traitant de l'Espagne coloniale reste, dans le roman, continuellement susceptible d'une autre analyse, d'une autre histoire. Célestine utilise d'ailleurs l'analyse comme un moyen d'approcher singulièrement la façon dont s'organisent et s'orientent les événements:

Elle qui au milieu des événements tient à maintenir la distance de la narration, assumant la nécessité d'analyser, plus que les faits eux-mêmes, la manière dont les faits s'extériorisent et sont liés entre eux... (t. II: 364)

Elle cherche, derrière ce qui est déclaré et extériorisé, les pensées et les contenus latents. À partir d'expériences oniriques relatives à des événements s'étant produits essentiellement au cours des XVe et $\mathrm{XVI}^{\mathrm{e}}$ siècles, Célestine tente d'analyser les rêves des trois bâtards, dont Pollo, puisqu'ils sont en lien direct avec l'histoire qu'elle tente de comprendre; ils la mettent sur une scène différente qui est à même d'en illustrer et d'en éclairer de nouvelles dimensions. L'analyse du contenu manifeste des songes lui permet de remonter à des sources plus profondes et cachées de l'histoire qui se trouvent dans l'esprit du dormeur. Si l'analyse conduite par les personnages peut parfois prétendre à un délire, de nombreuses situations démontrent toutefois qu'elle correspond davantage à une enquête minutieuse sur le passé, soutenue par des éléments de fragmentation, d'introspection et d'inflation verbale qui cherchent à fouiller et déterrer des vocables cachés.

\section{LECTURE DES PALIMPSESTES}

C'est un point d'originalité du roman que d'avoir su intégrer la mémoire de la découverte des Amériques 
en exposant le passé non plus à une synthèse ou à une reconstitution, mais à des entreprises de «dynamitages» et de fouilles mnésiques. La survivance et l'archéologie psychique que le passé engage composent ainsi une nouvelle conception de la mémoire cavée par une persistance des traces. Le roman propose l'idée que quelque chose se tapit infiniment sous ce qui est apparent et révélé. De fait, la difficulté à retrouver l'objet manquant (marquant), de l'avoir sur le bout de la langue comme d'autres l'ont sous les pieds, est sans cesse éprouvée par les personnages. Et à la différence de l'histoire qui est discours et connaissance, la mémoire, telle qu'elle est traitée par Fuentes, se dérobe toujours à une prise totalitaire, à une totale compréhension. Elle est un rappel de la défaillance du savoir pour des personnages qui, loin de maîtriser l'héritage duquel ils adviennent, s'y trouvent assujettis. Ne s'engageant pas sciemment dans une recherche du passé, c'est donc le passé qui vient les retrouver depuis leur rêve récurrent. De manière impromptue, car inspirée de la façon dont les pérégrins se déplacent dans le monde abymé du roman, les souvenirs s'avancent du fond de la mémoire ainsi que des ondins pris par la fantaisie de venir faire un tour tantôt chez l'un, tantôt chez l'autre. Les personnages eux-mêmes ne savent pas exactement d'où ils viennent, de quel temps, de quel fleuve ou de quel marais, de quel recoin de la mémoire ils émergent et refluent. Ils sont simplement remontés du fond de la vase, du fond d'une mémoire oublieuse. Leur mémoire est essentiellement affective. Elle est touchée par des pans douloureux du passé qui, comme dans le cas exemplaire de la petite Célestine, n'est pas le leur: «Les lèvres peintes, en revanche, lui apparaissaient comme une plaie, comme si en elles s'étaient retrouvés le temps de nouveau impitoyable, la douleur et l'humiliation» (t.I: 421). Le passé leur est imputé, transmis comme une charge. C'est ce qui amènera Célestine, dans le texte original, à s'exprimer sous le mode discursif de l'indirect libre. Elle parle, maintenant adolescente, à la vieille Célestine qui, «sans aucune mémoire, avait transmis toute son expérience et son savoir à l'autre» (t. II: 170), à elle.
Je me souviens de tout. J'ai vécu en pensant à toi, mère. Tu m'as laissé ta mémoire en même temps que tes baisers et tes caresses. J'ai grandi avec deux mémoires dans un seul corps. Et celle que tu m'as laissée était plus profonde que la mienne propre, car avec celle-là j'ai d̂u viure en silence pendant vingt ans, mère, sans pouvoir parler avec quiconque de ce que je me remémorais.

Aussi les propos de la petite Célestine, au sujet de cette mémoire vécue en silence qui l'a en quelque sorte temporisée, creusée, aident-ils à concevoir, voire à déchiffrer, les signes qui marquent ses lèvres et qui la distinguent singulièrement des autres personnages du roman. Par elle, la situation mnésique se précise, car, dans Terra Nostra, «la bouche est la mémoire» (t. II: 93), elle est ce qui pourrit sur les lèvres et résiste à l'effacement par des moyens cryptés. La mémoire espère son temps ( «tu sauras et tu diras ce que j'ai su et dit, je saurai et dirai, à temps, comme il me l'a dit, fais-le à temps» [t. II: 90]), de même qu'elle attend son protagoniste et son rêveur-interprète. La jeune fille attendait Pollo sur le pont, elle attendait que les trois frères ( «ils se rêvent tous les trois en même temps te dis-je, un rêve circulaire, infini» " [t. II: 158]) rêvent d'elle, de son histoire. Ainsi, lorsque sa mère symbolique, qui aura été impuissante à agir sur le déroulement des événements, trahie par l'histoire, lui lègue ses «lèvres blessées", elle lui révèle également ceci: «je te transmets ma voix, je te transmets ma bouche, je te transmets mes blessures, ma mémoire est sur tes lèvres» (t.II: 89). Ce qui est transmis à la petite Célestine est une blessure, une souffrance encore vive qui n'a pas trouvé le moyen de cautériser; elle reste comme une plaie sur les lèvres. Cette transmission approfondit et mine complètement le personnage puisqu'elle traduit la situation d'une vieille dame au passé douloureux, dont le témoignage s'accompagne aussi d'une éclipse mnésique. La dame a effectivement atteint une telle lourdeur et profondeur de vécu et de souvenirs que nous pourrions dire, à la suite du documentaire Sans soleil de Chris Marker, qu' «une mémoire totale est aussi une mémoire anesthésiée». Célestine transmet ses souvenirs à la petite en se 
vidant littéralement de sa mémoire, en la donnant complètement à l'autre qui s'en trouve assaillie et par là même chargée. Ce transfert mnésique renvoie également à la logique du temps passé œuvrant sur le territoire parisien abordée sous la figure d'un terrorisme temporel. L'amplitude du temps occupe l'espace du texte en allant jusqu'à désinvestir celui-ci de toute spécificité territoriale ou physique. L'histoire racontée dans Terra Nostra ne s'enracine pas dans une géographie, elle en tire tout au plus un motif exploité à des fins de transmission, de durée. Que ce soit par Célestine junior ou senior, Pollo, Ludovico ou encore Pedro, que ceux-ci se trouvent à Mexico, au Vietnam, en Italie, à Paris ou en Espagne, ce que l'ensemble des éléments poétiques exprime est la volonté de faire survivre le passé. En ce sens, la mémoire apparaît comme une faculté de transmission et de communication entre les temps et les personnages. La mise en évidence des aspects phénoménologique et empirique de la mémoire offre celle-ci comme une médiatrice du temps passé et du temps qui passe; la mémoire est du temps passé à l'autre. Elle est un incontestable don de temps dans le roman. Parmi les lectures que fait Ludovico dans la bibliothèque du Maestro Valerio Camillo, il trouve aussi, dans un livre «de Platon, les passages dans lesquels Socrate parle de la mémoire comme d'un don» (t.II: 113). La mémoire, comme l'exprime la relation particulière des deux Célestine dans le roman, est un legs: «je te lègue mes lèvres blessées, en elles ma mémoire [...]» (t.II: 89). Elle permet la survivance de souvenirs, de vies et d'histoires particulières, et ce, en dépit du fait qu'elle admette la perte. Car, dans Terra Nostra, révéler le passé généralement traumatisant équivaut aussi, comme l'exprime la situation de la vieille Célestine, à le léguer jusqu'à en perdre la mémoire. Cette transmission est hémorragique, elle circule certes, mais en vidant tour à tour le vaisseau mnésique qui l'innervait. Du coup, le traitement de la mémoire dans le roman permet d'induire un processus dynamique de transmission, de transmigration et de propagation. Son mouvement s'apparente à celui d'une épreuve de relais qui fait le pont entre ce qui a déjà été tracé, se retrace et doit être ultimement tracé. Relative à du passé, la mémoire est surtout ce qui passe à travers le temps. Le rêveur, qu'il soit Pollo, Philippe ou Célestine, est le mandataire de ces déplacements temporels qui l'amènent à se mouvoir de manière diachronique dans le récit abymé de l'histoire. Il est un dévaleur de temps et de mondes en palimpsestes dont il cherche les rhizomes. Rêveur étymologique, fureteur d'origines? Quoi qu'il en soit, l'opération archéologique s'opère à son insu. Même s'il perçoit une étrangeté relative à son expérience, il n'a pourtant pas conscience de rêver. Et c'est en cela qu'il se distingue de l'historien, car il est tout à fait inconscient du temps manipulé. Sa fonction est quasi archéologique: il passe à travers le temps pour y tomber chaque fois un peu plus: «si le temps est un chasseur, en cet instant précis il me perça de sa flèche; blessé, je tombai» (t. I: 747).

Puisque le souvenir transmis renvoie à un passé de douleur, d'assimilation et d'indignation, il suscite chez son dépositaire une sensation de chute et d'affalement. Celui qui subit la mémoire de ce temps en reste blessé, stigmatisé. Tous les enfants qui peuplent et hantent le roman sont des apatrides et des orphelins d'une Muse crevée, morte en couches. Avec la colonisation, c'est tout un héritage, un temps, qui a été suspendu, voire supprimé. Quelque chose ensuite s'est poursuivi, mais toujours escorté par une charge insondable d'oubli et une recherche douloureuse du sens. Or, malgré la tentative de la petite Célestine de se frotter la bouche avec la main, «le réseau de cicatrices ne s'effaçait pas» (t. II: 85). De même que les frères bâtards ne peuvent se défaire des rêves qui hantent leur sommeil, dont certains sont «induits, d'autres partagés» (t. I: 232), Célestine vit avec une seconde mémoire indélébile en elle. En confiant avoir "grandi avec deux mémoires dans un seul corps" (t. II: 165), elle pose aussi les jalons d'une poétique de la mémoire qui suppose et supporte une cohabitation des mondes et des vécus. Mais, encore, pour revenir à cet extrait où elle parle à sa mère amnésique alors incapable de la reconnaitre, ce qui retient l'attention est que l'adjonction d'une mémoire l'ayant laissée avec 
deux bouches («une bouche devait prononcer les paroles de ce temps; l'autre celles d'un temps oublié» [t. I: 48]) l'a aussi creusée. Rappelons-nous qu'elle traduit son impression d'avoir été abandonnée avec une mémoire "plus profonde» que la sienne propre. Ainsi, la mère Célestine ne lui a pas seulement légué un «réseau de cicatrices» sur la bouche, elle lui a également transmis une relation personnelle et sentimentale avec un temps qui la précède et la dépasse. Représentée, la mémoire pourrait donc aisément se laisser esquisser par les contours d'un foyer hanté. Dès lors, elle ne serait pas le lieu d'un propre, mais constituerait plutôt un espace parcouru et franchi par un «savoir atavique» (t. I: 21) et de multiples transmissions.

Aussi les lèvres cryptées de Célestine, les rêves des frères identifiés par une croix de chair et les yeux crevés de Ludovico constituent-ils une réserve de mémoire et de mots: "Le rêveur a une autre vie: la veille. L'aveugle a d'autres yeux: la mémoire" (t.II: 136). Une trace, un reste du passé résiste continûment à l'effacement et vient plier la surface homogène des faits. Cette trace fait signe depuis le passé dont elle n'est jamais revenue, marquée par un retard et un obstacle. En effet, elle joue sur l'amplitude et sur la possibilité d'être représentée et présentifiée, c'est-à-dire d'être rendue à la présence. Ainsi, même si, dans le cas de Célestine, la mémoire peine à s'actualiser et à se verbaliser, elle reste néanmoins là, en pleine surface, sur ses lèvres. La mémoire silencieuse marque ses lèvres et rend visibles les traces du refoulement. Ses lèvres stigmatisées constituent un véritable symptôme. Car c'est à partir de ce qui reste du passé, de ses traces, que les narrateurs tenteront de rappeler et de rêver ce qui a pu se passer. De ce fait, nous pourrions résumer le projet et l'esthétique du roman par l'idée de «tumulte anachronique», mise en place par Jacques Rancière dans Les Mots de l'histoire: «seul parle celui qui parlerait. Et non pas, surtout pas, celui qui parlait. Le conditionnel - l'anti-imparfait- de la parole en réserve, de la parole au tombeau" (2000: 119). Tous aussi poreux les uns que les autres, les personnages sont constamment traversés et habités par une parole du conditionnel et de l'anti-imparfait. Leur esprit constitue une loge pour tous les humiliés de l'histoire qui refluent à travers eux. Ils sont en quelque sorte les ventriloques d'une parole du tombeau et du désir. En eux, plusieurs événements du passé trouvent un espace de réfraction. Ou pour le dire autrement: dans Terra Nostra, le mort ${ }^{4}$ hante le vif et le rend cafouilleux. Car de même que le dit Barthes à propos de Michelet, il importe quelquefois de mettre en place un monde de représentation au sein duquel «les morts puissent se remettre à parler» (1965: 92), pour qu'enfin des mots gardés en suspens puissent trouver leur juste expression.

À plusieurs reprises dans le roman, les personnages se trouvent affectés et traversés par des réminiscences qui ne se réfèrent pas uniquement à leur seul champ d'expériences:

Dans la mémoire de ses ancêtres (je ne retins que ces images; elles s'infiltrèrent dans ma mémoire, déjà infiltrée par les souvenirs de mon père) défilaient comme des fantômes diurnes les cités pestilentielles, les guerres et les invasions [...]. (t. I: 209)

L'atavisme comme hypothèse de lecture du temps dans Terra Nostra évolue en parallèle avec l'exergue de Cernuda:

Ardente, déguenillée, c'est elle, la marâtre

Originaire. Dont tant, comme toi, sont

Douloureux et par elle dolents. 5

Cette marâtre originaire, pour reprendre l'expression du poète espagnol, rend les personnages douloureux et dolents. Pérégrin confie oublier "parce que se souvenir est douloureux [et il peine ainsi à se] donner la force d'accepter la douleur de la mémoire" (t.I: 737). Symbole de la Mémoire, elle est une mauvaise mère qui transmet la douleur à ses fils en les plaçant dans une filiation du souvenir déçu et terrifiant ( Je n'ai jamais très bien compris; seules me restèrent, comme je te le disais, certaines images qui toutes me parlaient de l'effondrement d'un monde» [t. I: 209]). De même, Ludovico est interpellé par la figure de Mnémosyne lors de ses lectures dans l'antre de Valerio: 
Socrate parle de la mémoire comme d'un don: c'est la mère des Muses, et dans toute âme il y a une part de cire dans laquelle se grave l'empreinte de nos pensées et perceptions. (t. II: 113)

Il y aurait à se demander, à la suite de l'événement colonial, des tromperies fourbes, des guerres et des catastrophes qui sillonnent Terra Nostra, si le roman ne présente pas la mémoire comme une souvenance et une inscription de la marge ( Pollo observa avec fascination la calligraphie des lèvres qui venaient de lui parler» [t. I: 48]), de l'absence, voire de la fosse. Testament originaire et inscriptible, la mémoire fuentésienne, ficelle pérenne, s'offrirait alors comme un moyen de communication des diverses résistances et déceptions historiques à travers le temps.

Communauté des morts, des disparus et des trahis, elle apparaît, sous ses atours cryptés de croix de chair entre les omoplates et de calligraphies sur les lèvres, comme une promesse en attente de son expression. Ce n'est qu'en attendant son tour, son accomplissement, que la mémoire terra nostrienne survit comme un stigmate sur les lèvres de la jeune fille et trouve à se symboliser dans le rêve. En espérant le moment opportun, Célestine, Ludovico, Pedro et les trois fils bâtards (les fils bâtards de la mémoire originaire?) rêvent. Ils rêvent du temps où leur rêve sera réalité et leur mémoire, histoire. Et c'est parce qu'elle est rêvée et qu'elle attend son réveil, qu'il est possible de penser que leur mémoire «évoquait des souvenirs très lointains ${ }^{6}$, comme un rêve [...]» (t.I: 343), souvenirs d'un originaire, pourrait dire Jung.

\section{CONCLUSION}

La mémoire a un statut particulier dans le roman de Fuentes. Elle est l'élément du réel qui compose l'apport référentiel du rêve mais qui, par les contingences et les mécanismes du procès onirique, se retrouve sans cesse déplacée vers un possible. Loin d'avoir tenté de retracer le passé à petits pas - sorte de mouvement récriminatoire et débiteur à l'égard du passé davantage disposé à évoquer ce qui aurait $d \hat{u}$ être-, Fuentes a proposé une histoire de ce qui aurait pu être. La stratégie fuentésienne est investie d'un rapport singulier du rêve au temps. Elle s'amuse ainsi, partant du fait que le désir marque la mémoire et que le rêve "est un accomplissement de désir", à produire une histoire redevable autant du passé que du futur; éléments prégnants de l'actualité du sujet.

Nous avons vu que si le rêve engage le sujet vers son archive, vers sa tablette de cire, il lui donne aussi l'élan de son illusion: il le met en face de ses désirs et de ses conflits. Le sens du roman résiderait donc dans une recherche temporelle irréductible à l'événement passé, puisque polarisée aussi par tout type d'avènement. Le rêve permet d'évoquer des images, de passer d'une image à une autre par le biais d'une suture imaginaire. A priori dépourvue de liens, la structure temporelle du roman les acquiert grâce au rêve, qui permet de créer une logique en abîme entre le monde parisien du XXe siècle et l'Espagne médiévale et, surtout, entre l'Espagne coloniale et l'Amérique décimée. Ce qui s'y discerne est l'idée matricielle d'un futur capable de retours sur le passé et d'un passé d'effets sur le futur; l'un révélant l'autre dans un infini ballet. Il y a ainsi une relation réversible entre ce qui a été présent (civilisation colombienne agressée et décimée) et ce qui le sera vraisemblablement. Et puisque c'est sur «les orifices du temps, durant les obscurs instants de vide où le passé tenta d'imaginer le futur» (t.I:481) que s'élabore le roman, il a été possible de montrer que le rêve pouvait induire la mémoire. 


\section{NOTES}

1. Je renverrai dorénavant à l'ouvrage de C. Fuentes en n'indiquant que le tome, suivi du numéro de page.

2. J. Rancière, 2000: 67.

3. À ce propos, voir J. Altounian, 1990 et 2000.

4. Mort est celui dans Terra Nostra qui a disparu, celui dont la trace a été effacée à tel point qu'il faille aujourd'hui recourir à l'imagination pour lui ouvrir un espace dans le champ de représentation du passé. 5. Ardiente y andrajosa. Es ella, la madrastra / Original de tantos, como tú, dolidos/ es ella y por ella dolientes. / Cernuda, Ser de Sansueña.

6. Dans son essai sur Freud et Nietzsche, P.-L. Assoun note que le rêve constitue une expérience archaïque. Sur les bases de Nietzsche, qui affirme que "dans le rêve continue à s'exercer cette partie antique de l'humanité" (1980: 194) et que "le rêve nous reporte dans de lointains états de la civilisation humaine et nous met en main un moyen de mieux les comprendre" (ibid.), Assoun conçoit le rêve comme un moyen régressif de voyager parmi les sédiments de la mémoire. "Le rêve, écritil, est un voyage de retour quotidien aux origines mentales de l'espèce, par le biais de la mémoire" (ibid.: 191). Fondamentalement, la notion d'archaïque dans le roman est investie d'un mobile d'exploration de la mémoire. Car il se trouve que c'est par le rêve que les personnages remontent aux souvenirs d'expériences à la fois antérieures à la découverte de l'Amérique et contemporaines.

\section{RÉFÉRENCES BIBLIOGRAPHIQUES}

Altounian, J. [1990]: Ouvrez-moi seulement les chemins d'Arménie: un génocide aux déserts de l'inconscient, Paris, Les Belles Lettres:

[2000]: La Survivance: traduire le trauma collectif, Paris, Gounod. Assoun, P.-L. [1980]: Freud et Nietzsche, Paris, P.U.F.

BARTHEs, R. [1965: Michelet par lui-même, Paris, Seuil.

FuENTES, C. [1979]: Terra Nostra, tomes I et II, Paris, Gallimard, coll. "Folio".

MARKER, C. [1982]: Sans soleil (documentaire), Paris, Argos Films. RANCIÈRE, J. [2000]: Les Mots de l'histoire. Essai de poétique du savoir, Paris, Seuil. 